\title{
Buchbesprechung
}

\section{Die ärztliche Begutachtung}

\section{Fritze J, Mehrhoff F}

8., vollständig überarbeitete und aktualisierte Auflage, Heidelberg: Springer Verlag 2012,

900 S. m. 90 Abb. und 200 Tab., 179,95€

ISBN 978-3-642-21080-8

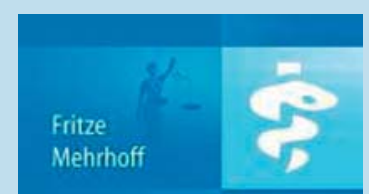

Die ärztliche Begutachtung

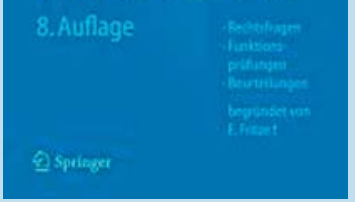

Ein Klassiker neu aufgelegt - nach der ersten Auflage von Eugen Fritze von 1982 liegt nun die achte Auflage in vollständiger Überarbeitung vor. Zweifelsfrei und unangefochten das deutschsprachige Referenzwerk der ärztlichen Begutachtung, erhebt es den Anspruch, „nahezu alle Gebiete der Medizin“ "zu erfassen. Diesem Anspruch liegt die Auffassung zugrunde, dass jeder Gutachter Begutachtungen aus verschiedenen Fachgebieten zusammenführen können muss. Das ist ein sehr hoher Anspruch, der wohl meistens nicht in diesem Umfang erfüllt sein muss. Dennoch ist es sehr nützlich, eine Gesamtübersicht und somit eine zentrale Referenz vor sich zu haben.

Bereits im Vorwort werden einige Klarstellungen hinsichtlich der unterschiedlichen Bedeutung von GdB, MdE und GdS vorgenommen, die dem Leser klarmachen, dass es mit der Begutachtung kein Leichtes ist. Die ersten rund 100 Seiten befassen sich daher neben allgemeinen Grundlagen mit rechtlichen Fragen, die nächsten rund 25 mit Arztrecht. Die folgenden rund 100 Seiten umfassen Funktionsprüfungen der Organe. Ca. 13 Seiten nimmt dabei die pneumologische Funktionsdiagnostik ein, kompetent vorgetragen von prominenten Vertretern unseres Faches. Rund 550 Seiten bilden das medizinische Kernstück des Buches, die Darstellung der Krankheiten, und erneut sind etwa $10 \%$ den pneumologischen Krankheiten gewidmet. Natürlich sind auch Kapitel zu chemischen Einwirkungen, Arzneimittel- und Medizinprodukteschäden, physikalischen Einflüssen, iatrogenen Schäden sowie - tatsächlich - komplementärer und alternativer Medizin eingeschlossen. Ein Kapitel widmet sich der Fahreignung. Natürlich darf auch eines zum Gutachterhonorar nicht fehlen, das aber mit ca. sechs Seiten denkbar knapp ausfällt.

Ein Riesenwerk also. Dennoch ist es gelungen, den Text sowohl gut lesbar als auch tauglich zu halten als Nachschlagewerk. Die Darstellung ist didaktisch durchweg gut gelungen, die Texte sind hinreichend ausführlich, ohne zu lang zu geraten.

Selbst ein solches Werk kann natürlich eine fachgebundene Referenz nicht ersetzen, sondern nur ergänzen. Insofern werden zumindest die Organkapitel überwiegend als Referenz nachgelesen werden. Dessen ungeachtet gebührt diesem Werk sein fester Platz in der Hand eines jeden Gutachters, der den Anspruch stellt, wissenschaftlich fundiert und praktisch informiert Gutachten zu verfassen, die einem Einspruch standhalten können.

Prof. Dr. med. Santiago Ewig, Bochum 